\title{
Staging of tau distribution by positron emission tomography may be useful in clinical staging of Alzheimer disease
}

Kenta Aohara, Shinobu Minatani, Hiroko Kimura, Jun Takeuchi, Akitoshi Takeda, Joji Kawabe, Yasuhiro Wada, Aya Mawatari, Yasuyoshi Watanabe, Hitoshi Shimada, Makoto Higuchi, Tetsuya Suhara, Yoshiaki Itoh

\begin{tabular}{|c|l|}
\hline Citation & Neurology and Clinical Neuroscience. 8(2); 61-67 \\
\hline Issue Date & $2020-03-01$ \\
\hline Type & Journal Article \\
\hline Textversion & Author \\
\hline Relation & $\begin{array}{l}\text { This is the peer reviewed version of the following article: Neurology and } \\
\text { Clinical Neuroscience. Volume8, Issue2. Pages } 61-67 ., \text { which has been } \\
\text { published in final form at https://doi.org/10.1111/ncn3.12366. This article } \\
\text { may be used for non-commercial purposes in accordance with Wiley Terms } \\
\text { and Conditions for Use of Self-Archived Versions. }\end{array}$ \\
\hline DOI & $10.1111 /$ ncn3.12366 \\
\hline
\end{tabular}

Self-Archiving by Author(s)

Placed on: Osaka City University Repository

AOHARA K., et al. (2020). Staging of tau distribution by positron emission tomography may be useful in clinical staging of Alzheimer disease. Neurology and Clinical Neuroscience. 8, 61-67. DOI:10.1111/ncn 3.12366 


\title{
Neurology and Clinical Neuroscience
}

\author{
ORIGINAL ARTICLE
}

\section{Staging of tau distribution by positron emission tomography may be useful in clinical staging of Alzheimer disease}

Kenta Aohara ${ }^{1}$, Shinobu Minatani ${ }^{1}$, Hiroko Kimura ${ }^{1}$, Jun Takeuchi ${ }^{1}$, Akitoshi Takeda ${ }^{1}$, Joji Kawabe' ${ }^{2}$, Yasuhiro Wada ${ }^{3}$, Aya Mawatari ${ }^{3}$, Yasuyoshi Watanabe ${ }^{3}$, Hitoshi Shimada ${ }^{4}$, Makoto Higuchi ${ }^{4}$, Tetsuya Suhara ${ }^{4}$, Yoshiaki Itoh ${ }^{1}$

${ }^{1}$ Department of Neurology and ${ }^{2}$ Department of Nuclear Medicine, Osaka City University Graduate School of Medicine, Osaka, Japan;

${ }^{3}$ RIKEN Center for Biosystems Dynamics Research, Kobe, Japan;

${ }^{4}$ Department of Functional Brain Imaging Research (DOFI), Clinical Research Cluster, National Institute of Radiological Sciences (NIRS), National Institutes for Quantum and Radiological Science and Technology (QST), Chiba, Japan

\section{Correspondence}

Yoshiaki Itoh

Department of Neurology, Osaka City University Graduate School of Medicine, Asahicho 1-4-3, Abenoku, Osaka City, Osaka Prefecture, 545-8585, Japan

E-mail:y-itoh@med.osaka-cu.ac.jp

\section{Short title}

Staging of Alzheimer disease by Tau PET 


\section{Abstract}

Background: Clinical staging of Alzheimer disease (AD) may help develop novel treatment in the early stage.

Aim: We measured the accumulation of amyloid beta $(\mathrm{A} \beta)$ and tau with positron emission tomography (PET) in patients with $\mathrm{AD}$ to explore its utility for clinical staging. Methods: Six patients with AD, two patients with mild cognitive impairment (MCI), and 12 healthy controls (HC) were studied. A $\beta$ and tau accumulation was evaluated with $\left.{ }^{11} \mathrm{C}\right] \mathrm{PiB}$ and $\left[{ }^{11} \mathrm{C}\right] \mathrm{PBB} 3$, respectively.

Results: PBB3-PET showed that two cases were in stage I-II (Braak stage), one case was in stage III-IV, and three cases were in stage V-VI. PiB-PET showed that all cases were in stage C. The duration of the disease correlated positively with PBB3 staging but not with PiB staging. The performance on the Mini-Mental State Examination (MMSE) tended to decrease with advancing of PBB3 stage but not with PiB stage. PBB3 SUVR and PiB SUVR in all AD signature areas including the parahippocampal gyrus were significantly higher in AD than in HC. A decrease in MMSE is correlated with increases in PBB3 and PiB. Increase in PBB3 started with decrease in MMSE whereas increase in PiB was already observed in the point of highest MMSE, indicating amyloid is already accumulated in the earliest stage.

Conclusions: The expansion of tau distribution from the parahippocampal gyrus to the cerebral cortex was observed with advancing $\mathrm{AD}$, whereas $\mathrm{A} \beta$ distribution was already advanced in the earliest stage. PBB3 may be useful in determining stages in AD based on tau distribution.

\section{Key words}

amyloid PET, dementia, parahippocampal gyrus, PBB3, PiB, 


\section{Introduction}

The classical amyloid hypothesis of Alzheimer disease (AD) suggests that polymerization of amyloid beta $(\mathrm{A} \beta)$ is the first step to the common pathway of neurodegeneration. ${ }^{1}$ Tau accumulation and neuronal dysfunction may follow the $A \beta$ accumulation..$^{2}$ However, this sequence was mainly confirmed in hereditary $\mathrm{AD},{ }^{3}$ and little is reported on sporadic $\mathrm{AD}$. We previously reported that a mild amount of tau accumulates age-dependently in healthy controls and may trigger robust $\mathrm{A} \beta$ accumulation and further tau accumulation in $\mathrm{AD}$ development. ${ }^{4}$ Furthermore, the failure of many clinical trials targeting $\mathrm{A} \beta$ in already developed $\mathrm{AD}$ indicates that not A $\beta$ but tau may be the main worsening factor after onset. ${ }^{5}$ Thus, tau may be involved in the progress of $\mathrm{AD}$ and has become a novel target of $\mathrm{AD}$ treatment. ${ }^{6}$

The pathological accumulation of $\mathrm{A} \beta$ as well as neurofibrillary tangle (NFT) was suggested to spread from the limbic system to the neocortex, whereas few clinical data on such spread based on $\mathrm{AD}$ staging were reported.7,8 $\mathrm{A} \beta$ is already accumulated at $\mathrm{AD}$ signature areas in early $\mathrm{AD}$ or even in mild cognitive dementia (MCI) due to AD.9-11 The spreading pattern of $A \beta$ has been scarcely reported along the stages of advancement of $\mathrm{AD}^{9-11}$. In addition to $\mathrm{A} \beta$, we measured the accumulation of tau with a radiopharmaceutically significant tracer, $2-\left[(1 \mathrm{E}, 3 \mathrm{E})-4-\left[6-\left[{ }^{11} \mathrm{C}\right]\right.\right.$ methylamino $]$ 
pyridin-3-yl]buta-1,3-dien-1-yl]-1,3-benzothiazol-6-ol ([11C]PBB3), for positron emission tomography (PET) 12-14 in patients with various durations of $\mathrm{AD}$ and judged the stage of tau distribution based on the standard pathological staging by Braak. ${ }^{7}$

\section{Materials and Methods}

Ethical approval. All procedures performed in studies involving human participants were approved by the Institutional Research Ethics Committee of Osaka City University Graduate School of Medicine (IRB\# 3009 and 1735) and were in accordance with the 1964 Helsinki declaration and its later amendments.

Informed consent. Written informed consent was obtained from all participants or from close family when the participants were cognitively impaired.

Healthy Controls. Healthy controls (HC) without history of brain disorders or subjective abnormality were openly recruited as candidates. A board-certified neurologist interviewed them and obtained detailed medical histories including alcohol consumption. All candidates took physical examination covering vital and cardiopulmonary systems. Thorough neurological examinations, including general 
cognitive function tests, were performed by the same neurologist. The Mini-Mental State Examination (MMSE) and the revised Hasegawa Dementia Scale (HDS-R) were scored by qualified clinical psychologists (MA, NK). Blood samples were collected to evaluate general physical condition, including thyroid function and vitamin B1. Magnetic resonance images (MRIs) were also evaluated.

The exclusion criteria were as follows: 1) history of any brain diseases, brain surgery, or head trauma requiring hospitalization; 2) high risk for cerebrovascular diseases, including poorly controlled diabetes, dyslipidemia, and high blood pressure above the recommended level of standard guidelines; 3) any neurological findings suggesting brain disorders; 4) cognitive test [MMSE, HDS-R, and RBMT (Rivermead Behavioral Memory Test)] indicating border-zone or worse level; and 5) MRI lesions including asymptomatic lacunas (less than $15 \mathrm{~mm}$ in diameter, high in T2, FLAIR and low in T1), severe white matter lesion (more than 1 in Fazekas grade ${ }^{15)}$, more than 4 cerebral microbleeds, and atrophy beyond average for their age by visual inspection; and 6) amyloid PET imaging was employed for the exclusion of enrollment. No other biomarkers were examined in the HC group.

AD and $\mathbf{M C l}$. Cases with $\mathrm{AD}$ and $\mathrm{MCI}$ due to $\mathrm{AD}$ were recruited from patients attending 
the outpatient clinic of Osaka City University Hospital. Medical history, neurological findings and general blood tests were evaluated by neurologists specializing in dementia. MMSE and HDS-R were scored by qualified clinical psychologists. An MRI of the brain including coronal section was taken.

The diagnostic criteria of MCI due to $\mathrm{AD}$ by the National Institute on Aging-Alzheimer's Association workgroups was employed for the diagnosis of MCI. ${ }^{16}$ To better understand the role of tau compared with $\mathrm{A} \beta$ in the development of $\mathrm{AD}, \mathrm{MCI}$ was diagnosed regardless of the findings of amyloid PET imaging. For the diagnosis of AD, IWG-2 criteria for typical AD was employed with amyloid PET as an in vivo evidence of $\mathrm{AD}$ pathology. ${ }^{17}$ Apparent familial $\mathrm{AD}$ was not included in the present study.

The same exclusion criteria applied for $\mathrm{HC}$ were applied for $\mathrm{AD}$ and MCI to exclude confounding diseases such as diabetes, dyslipidemia and hypertension.

Disease duration of $\mathrm{AD}$ was defined as a period from the onset of dementia to the time of the present imaging study. The onset was determined as a converting time in case of MCI or retrospectively speculated at the initial visit for early AD. No cases without proper medical records on the onset were involved.

PET data acquisition. $\left.{ }^{[11} \mathrm{C}\right] \mathrm{PBB} 3$ and $\left[{ }^{11} \mathrm{C}\right] \mathrm{Pittsburgh}$ compound-B $\left(2-\left[4^{-}\left(\left[{ }^{11} \mathrm{C}\right]\right.\right.\right.$ 
methylamino) phenyl]-1,3-benzothiazol-6-ol, $\left.\left[{ }^{11} \mathrm{C}\right] \mathrm{PiB}\right)$ were produced based on previously reported methods. ${ }^{13,18,19}\left[{ }^{11} \mathrm{C}\right]$ PBB3-and $\left[{ }^{11} \mathrm{C}\right] \mathrm{PiB}-\mathrm{PET}$ images were acquired with a Siemens Biograph16 scanner (Siemens/CTI, Knoxville, TN, USA) and with an Eminence-B PET scanner (Shimadzu Co., Kyoto, Japan), respectively. For tau imaging, [11C]PBB3 in the range of $370 \mathrm{MBq}$ (body weight $\leq 50 \mathrm{~kg}$ ) to $555 \mathrm{MBq}$ (body weight $\geq 70$ kg) was intravenously injected to the patient in a dimly lit room to avoid photoracemization. A 60-minute PET scan was performed in the list mode. The acquired data were sorted into dynamic data of $6 \times 10 \mathrm{~s}, 3 \times 20 \mathrm{~s}, 6 \times 60 \mathrm{~s}, 4 \times 180 \mathrm{~s}$, and $8 \times 300 \mathrm{~s}$ frames. For $\mathrm{A} \beta$ imaging, each subject received 400 to $500 \mathrm{MBq}$ of $\left[{ }^{11} \mathrm{C}\right] \mathrm{PiB}$ intravenously over 1 minute. After the injection, a static scan image acquisition was performed for 50 to 70 minutes. PET images for $\left[{ }^{11} \mathrm{C}\right] \mathrm{PBB} 3$ and $\left[{ }^{11} \mathrm{C}\right] \mathrm{PiB}$ were reconstructed by filtered back projection using a 4-mm full width at half maximum (FWHM) Hanning filter and a 5-mm FWHM gaussian filter with attenuation and scatter correction, respectively.

MRI acquisition. The MRI data were obtained with a 1.5- or 3-Tesla magnetic resonance scanner (MAGNETOM Avanto, Siemens Healthcare, Erlangen, Germany or Ingenia, Philips Healthcare, Best, The Netherlands). Three-dimensional volumetric acquisition of a T1- weighted gradient echo sequence (repetition time range/echo time range, 6.5 
$\mathrm{ms} / 3.2 \mathrm{~ms}$; field of view [frequency $\times$ phase], $240 \mathrm{~mm} \times 240 \mathrm{~mm}$; matrix, $256 \times 256$;

contiguous axial slices of $1.5 \mathrm{~mm}$ thickness) was performed.

Image processing. All images were processed using PMOD software version 3.7 (PMOD

Technologies Ltd., Zurich, Switzerland) ${ }^{20}$ and Statistical Parametric Mapping software

(SPM12, Wellcome Department of Cognitive Neurology, London, UK), operating on the MATLAB software environment (version R2016b; MathWorks, Natick, MA, USA)21. Acquired $\left[{ }^{11} \mathrm{C}\right] \mathrm{PBB} 3$ and $\left.{ }^{[11} \mathrm{C}\right] \mathrm{PiB}$ images were transformed into standard brain using PMOD, and then SUVR images were generated with the cerebellar cortex as a reference region using the frame summation of dynamic image for 30 to 50 minutes after

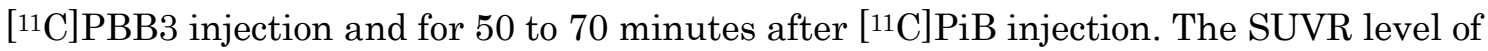
each volume of interest (VOI) was calculated in a manually set region of interest (ROI) as shown in Online Resource 1.

Each T1-weight MRI was segmented and anatomically normalized to MNI152 standard space (Montreal Neurological Institute, Montreal, QC, Canada) using SPM12 and Diffeomorphic Anatomical Registration Through Exponentiated Lie Algebra (DARTEL) ${ }^{22}$. The $\left[{ }^{11} \mathrm{C}\right] \mathrm{PBB} 3 \mathrm{SUVR}$ and $\left[{ }^{11} \mathrm{C}\right] \mathrm{PiB}$ SUVR images were co-registered to individual T1-weighted MRI using PMOD and then normalized to MNI space using the 
same parameters as those for MRI (flow fields method) with smoothing at 8-mm full-width-at-half-maximum.

Criteria for $A \beta$ accumulation. $A \beta$ accumulation was visually evaluated based on the Japanese Alzheimer's Disease Neuroimaging Initiative (J-ADNI) Visual Criteria for [11C]PiB-PET (J-ADNI_PETQC_Ver1.1) modified from ADNI PET core criteria ${ }^{23}$. The four regions selected for the assessment were the precuneus combined with posterior cingulate gyrus, frontal lobe, lateral temporal lobe, and lateral parietal lobe. When the accumulation in one of the four cerebral cortices was higher than that in the white matter just below the cortex, the case was determined as positive. When none of the four cortices had higher accumulation than the white matter, the case was confirmed as negative.

Voxel-wise analysis of $\left[{ }^{11} \mathrm{C}\right] \mathrm{PBB} 3$ and $\left[{ }^{11} \mathrm{C}\right] \mathrm{PiB}$. A voxel-wise comparison of ${ }^{\left[{ }^{11} \mathrm{C}\right] \mathrm{PBB} 3}$ and $\left.{ }^{[11} \mathrm{C}\right] \mathrm{PiB}$ accumulation data was performed between individual $\mathrm{AD}$ cases and the HC group by single subject analysis using SPM12. ${ }^{24}$ Age was included as a covariate. The threshold for significance was set at $P<0.05$ family-wise error (FWE)-corrected at cluster level following $P<0.01$ uncorrected at voxel level. 
Regional SUVR and cognitive impairment in AD. In each ROI, regional SUVR was compared between $\mathrm{HC}$ and $\mathrm{AD}$ with a Mann-Whitney $U$-test, and the significance level was $5 \%$, two-tailed test. In addition to each ROI, the non-weighted average SUVR in the $\mathrm{AD}$ signature regions (i.e., the lateral temporal cortex, frontal cortex, posterior cingulate gyrus, precuneus, and parietal cortex) was statistically compared between the two groups. The parahippocampal gyrus, which is pathologically known to have no or very limited accumulation of $\mathrm{A} \beta$, was not included in the $\mathrm{AD}$ signature region.

The correlation between the SUVR and MMSE evaluated at the same time in $\mathrm{AD}$ was statistically evaluated in each ROI as well as in the averaged $\mathrm{AD}$ signature regions by the Spearman's rank correlation coefficient. $P<0.05$ was regarded as statistical significance. Analyses were carried out using SPSS version 25 for windows (SPSS Inc., Chicago, IL, USA).

\section{Results}

Demographical data. Table 1 shows the demographical data of the AD, MCI, and HC groups. The age of patients in the $\mathrm{AD}$ group varied widely from 51 to 85 years old. Two patients with MCI were in their over 80 years of age. The age distribution of the HC 
group was similar to that of the $\mathrm{AD}$ group $[71.8 \pm 8.7$ years, mean \pm standard deviation (SD)]. In the AD group, either MMSE or HDS-R was below the cutoff level of dementia, whereas subjects in the MCI and HC groups had little or no deduction of points on the cognitive tests.

Braak's staging. In each patient with $\mathrm{AD}, \mathrm{PBB} 3$ as well as $\mathrm{PiB}$ accumulation was statistically compared with that of the HC group by the statistical parametric mapping method. Stage of PBB3 and PiB distribution was determined based on the pathological stage of NFT and amyloid, respectively, by Braak (Fig 1). The PBB3-PET study showed that two cases were in stage I-II (transentorhinal stage), one case was in stage III-IV (limbic stage), and three cases were in stage V-VI (isocortical stage); whereas, PiB-PET showed that all cases were in stage $\mathrm{C}$ (neocortical stage) (Table 1).

The duration of the disease correlated positively with PBB3 staging but not with PiB staging. MMSE tended to decrease with advancing of PBB3 stage, but not of PiB stage (Fig 2).

\section{Cognitive deterioration and tau/amyloid beta}

PBB3 SUVR and PiB SUVR in all AD signature regions as well as the parahippocampal 
gyrus were significantly higher in the $\mathrm{AD}$ group than in the $\mathrm{HC}$ group $(P<0.05)$ (Fig 3).

The decrease in MMSE was statistically significantly correlated with the increase in PiB $(P<0.05)$. A similar tendency was found in the decrease in MMSE and increase in PBB3, without statistical significance.

Of note is that the linear correlation between the decrease in MMSE and increase in PBB3 crossed the basal point, namely the SUVR level of the HC group, whereas the linear correlation between the decrease in MMSE and increase in PiB shifted higher and did not cross the basal point (Fig 3), indicating that amyloid is already widely distributed in the earliest stage of $\mathrm{AD}$. The shift in PiB was not observed in the parahippocampal gyrus.

\section{Discussion}

In the present PET study, expansion of tau distribution from the transentorhinal cortex to the cerebral cortex was observed with advancing cognitive severity of $\mathrm{AD}$, whereas $\mathrm{A} \beta$ distribution was already advanced in the earliest stage. These results suggest that PBB3 may be useful in determining stages in AD based on tau distribution, whereas $\mathrm{PiB}$ is helpful in early diagnosis of $\mathrm{AD}$. 
Similar report supporting our data has been recently published by Jack et $\mathrm{al}^{25}$.

They also reported two steps in developing $\mathrm{AD}$; i.e. first $\mathrm{A} \beta$ accumulation and later tau. However they failed to analyze spatial development of the pathological changes we reported in the present study.

We previously reported that tau accumulates with age in the region related to AD. ${ }^{4}$ This is in good accordance with the previous reports by Jagust WJ et al. regarding [18F]AV1451. ${ }^{26}$ In the present study, we found further accumulation of tau in accordance with cognitive deterioration, starting from the transentorhinal cortex and extending into neocortex.

A clinicopathological study already reported that MMSE was significantly correlated with NFT and neuron number but not with amyloid volume in CA1, the entorhinal cortex, and area 9.27 NFT and neuronal loss but not senile plaque in superior temporal sulcus were correlated with disease severity. ${ }^{28}$ Using novel tau tracer $[18 \mathrm{~F}] \mathrm{AV} 1451$ and classical A $\beta$ tracer $[18 \mathrm{~F}] \mathrm{AV} 45$, tau accumulation was strongly correlated and $\mathrm{A} \beta$ was only mildly correlated with cognitive decline in AD. ${ }^{29}$ These reports are quite consistent with the present findings that $\mathrm{A} \beta$ is already accumulated in the earliest stage of $\mathrm{AD}$ and continues to accumulate slowly thereafter.

A recent pathological analysis also reported widespread but exclusive cortical 
distribution of $\mathrm{A} \beta$ in the earliest stage of $\mathrm{AD} .{ }^{30}$ Utilizing the North American

Alzheimer's Disease Neuroimaging Initiative (ADNI) cohort, Palmqvist et al. reported that the earliest accumulation of $\mathrm{A} \beta$ was found within the default-mode network, including the posterior cingulate cortex, the precuneus, and the medial orbitofrontal cortex. ${ }^{31}$ The accumulation became widespread before the onset of dementia. ${ }^{31}$ Using the same ADNI data with tau PET by [18F]AV1451, Tosun et al. reported that early tau deposition was found in the inferior temporal and inferior parietal regions. ${ }^{32}$ The further deposition pattern varied with different cognitive performance and clinical outcome measures. ${ }^{32}$ These reports are in good agreement with the present findings that the spread of tau reflects cognitive deterioration in typical AD cases.

One of the drawbacks of $\left.{ }^{11} \mathrm{C}\right] \mathrm{PBB} 3$ is that it accumulates at the cerebral sinuses including the superior sagittal sinus and transverse sinus. The extent of accumulation varies between cases. Low accumulation can be eliminated by masking the accumulation at the sinuses, although high accumulation can affect apparent measurement of the cerebral cortex. In the present study, high accumulation neighboring the superior sagittal sinus and transverse sinus in the occipital lobe was considered as artifact.

The limited dynamic range of $\left[{ }^{11} \mathrm{C}\right] \mathrm{PBB} 3$ to detect tau accumulation also may 
overlook smaller lesion or milder accumulation. ${ }^{14}$ In the present study, increase in $\left.{ }^{11} \mathrm{C}\right]$ PBB3 accumulation at the parahippocampal gyrus and lateral temporal cortex, the regions usually involved in the earliest stage, was not detected in a case with AD. Possible overestimation of the aging effect on tau accumulation at these regions may additionally reduce the significance ${ }^{4}$. Further development of a more sensitive tracer is warranted.

Pathology was not confirmed in any case in the present study. There might be some gap between $\left.{ }^{11} \mathrm{C}\right] \mathrm{PBB} 3$ accumulation and pathological changes. Although the distribution of tau by $\left.{ }^{11} \mathrm{C}\right] \mathrm{PBB} 3$ in $\mathrm{AD}$ in the present study is consistent with the distribution of lesions in the previous pathological reports, further pathological confirmation is warranted. A greater number of subjects with $\mathrm{AD}$ should be studied to draw definite conclusions.

Acknowledgments: The authors thank Ms. M. Ando and Ms. N. Kotani (Osaka City University) for psychological tests. The present study was financially supported by the NIRS/QST grant (Japan Agency for Medical Research and Development 16768966), in which Osaka City University was involved as a collaborating facility.

Conflict of Interest: Authors H.S., M.H., and T.S. possess patent royalty of PBB3.

\section{References}

1. Selkoe DJ. The molecular pathology of Alzheimer's disease. Neuron 1991;6:487-98.

2. Lemere CA, Lopera F, Kosik KS, et al. The E280A presenilin 1 Alzheimer mutation 
produces increased AB42 deposition and severe cerebellar pathology. Nature Medicine 1996;2:1146.

3. Bateman RJ, Xiong C, Benzinger TLS, et al. Clinical and Biomarker Changes in Dominantly Inherited Alzheimer's Disease. New England Journal of Medicine 2012;367:795-804.

4. Kikukawa T, Saito H, Hasegawa I, et al. Localized Accumulation of Tau without Amyloid-beta in Aged Brains Measured with 11[C]PBB3 and [11C]PiB Positron Emission Tomography. Journal of Alzheimers Disease and Parkinsonism 2017;7:6.

5. Honig LS, Vellas B, Woodward M, et al. Trial of Solanezumab for Mild Dementia Due to Alzheimer's Disease. N Engl J Med 2018;378:321-30.

6. Li C, Gotz J. Tau-based therapies in neurodegeneration: opportunities and challenges. Nat Rev Drug Discov 2017;16:863-83.

7. Braak H, Braak E. Neuropathological stageing of Alzheimer-related changes. Acta Neuropathol 1991;82:239-59.

8. Schwarz AJ, Yu P, Miller BB, et al. Regional profiles of the candidate tau PET ligand 18F-AV-1451 recapitulate key features of Braak histopathological stages. Brain 2016;139:1539-50.

9. Grothe MJ, Barthel H, Sepulcre J, Dyrba M, Sabri O, Teipel SJ. In vivo staging of regional amyloid deposition. Neurology 2017;89:2031-8.

10. Thal DR, Attems J, Ewers M. Spreading of amyloid, tau, and microvascular pathology in Alzheimer's disease: findings from neuropathological and neuroimaging studies. J Alzheimers Dis 2014;42 Suppl 4:S421-9.

11. Veitch DP, Weiner MW, Aisen PS, et al. Understanding disease progression and improving Alzheimer's disease clinical trials: Recent highlights from the Alzheimer's Disease Neuroimaging Initiative. Alzheimers Dement 2018.

12. Shimada H, Kitamura S, Shinotoh H, et al. Association between Abeta and tau accumulations and their influence on clinical features in aging and Alzheimer's disease spectrum brains: A [11C]PBB3-PET study. Alzheimers Dement (Amst) 2017;6:11-20.

13. Maruyama M, Shimada H, Suhara $\mathrm{T}$, et al. Imaging of tau pathology in a tauopathy mouse model and in Alzheimer patients compared to normal controls. Neuron 2013;79:1094-108.

14. Ono M, Sahara N, Kumata K, et al. Distinct binding of PET ligands PBB3 and AV-1451 to tau fibril strains in neurodegenerative tauopathies. Brain 2017;140:764-80.

15. Fazekas F, Chawluk JB, Alavi A, Hurtig HI, Zimmerman RA. MR signal abnormalities at $1.5 \mathrm{~T}$ in Alzheimer's dementia and normal aging. AJR Am J Roentgenol 1987;149:351-6.

16. Jack CR, Jr., Albert MS, Knopman DS, et al. Introduction to the recommendations 
from the National Institute on Aging-Alzheimer's Association workgroups on diagnostic guidelines for Alzheimer's disease. Alzheimers Dement 2011;7:257-62.

17. Dubois B, Feldman HH, Jacova C, et al. Advancing research diagnostic criteria for Alzheimer's disease: the IWG-2 criteria. Lancet Neurol 2014;13:614-29.

18. Kimura Y, Ichise M, Ito H, et al. PET Quantification of Tau Pathology in Human Brain with 11C-PBB3. J Nucl Med 2015;56:1359-65.

19. Hashimoto H, Kawamura K, Igarashi N, et al. Radiosynthesis, photoisomerization, biodistribution, and metabolite analysis of 11C-PBB3 as a clinically useful PET probe for imaging of tau pathology. J Nucl Med 2014;55:1532-8.

20. Choi WH, Um YH, Jung WS, Kim SH. Automated quantification of amyloid positron emission tomography: a comparison of PMOD and MIMneuro. Annals of Nuclear Medicine 2016;30:682-9.

21. Gallivanone F, Della Rosa PA, Castiglioni I. Statistical Voxel-Based Methods and [18F]FDG PET Brain Imaging: Frontiers for the Diagnosis of AD. Curr Alzheimer Res 2016;13:682-94.

22. Ashburner J. A fast diffeomorphic image registration algorithm. Neuroimage 2007;38:95-113.

23. Jagust WJ, Bandy D, Chen K, et al. The ADNI PET Core. Alzheimers Dement 2010;6:221-9.

24. Maass A, Landau S, Baker SL, et al. Comparison of multiple tau-PET measures as biomarkers in aging and Alzheimer's disease. Neuroimage 2017;157:448-63.

25. Jack CR, Wiste HJ, Botha H, et al. The bivariate distribution of amyloid-beta and tau: relationship with established neurocognitive clinical syndromes. Brain 2019;142:3230-42.

26. Schöll M, Lockhart Samuel N, Schonhaut Daniel R, et al. PET Imaging of Tau Deposition in the Aging Human Brain. Neuron 2016;89:971-82.

27. Giannakopoulos P, Herrmann FR, Bussiere T, et al. Tangle and neuron numbers, but not amyloid load, predict cognitive status in Alzheimer's disease. Neurology 2003;60:1495-500.

28. Gomez-Isla T, Hollister R, West H, et al. Neuronal loss correlates with but exceeds neurofibrillary tangles in Alzheimer's disease. Ann Neurol 1997;41:17-24.

29. Koychev I, Gunn RN, Firouzian A, et al. PET Tau and Amyloid-beta Burden in Mild Alzheimer's Disease: Divergent Relationship with Age, Cognition, and Cerebrospinal Fluid Biomarkers. J Alzheimers Dis 2017;60:283-93.

30. Thal DR, Rub U, Orantes M, Braak H. Phases of A beta-deposition in the human brain and its relevance for the development of AD. Neurology 2002;58:1791-800.

31. Palmqvist S, Scholl M, Strandberg O, et al. Earliest accumulation of beta-amyloid 
occurs within the default-mode network and concurrently affects brain connectivity. Nat Commun 2017;8:1214.

32. Tosun D, Landau S, Aisen PS, et al. Association between tau deposition and antecedent amyloid-beta accumulation rates in normal and early symptomatic individuals. Brain 2017;140:1499-512. 


\section{Figure Legends}

Figure 1. Representative cases for Braak's staging of NFT (a) and senile plaque (b). In each case with AD, PBB3 (a) as well as PiB (b) accumulation was statistically compared with that of $\mathrm{HC}$ by the statistical parametric mapping method. No case was classified as stage A or stage B in Braak's staging of amyloid distribution (b).

Figure 2. Disease duration and Mini-Mental State Examination (MMSE) score at each stage in patients with $\mathrm{AD}$. (a), (b) indicate neurofibrillary change staging of PBB3, (c) and (d) indicate amyloid deposits staging of PiB.

Figure 3. Scatter plots show correlation between Mini-Mental State Examination (MMSE) and PBB3 SUVR (a, c) or MMSE and PiB SUVR (b, d) in AD

signature regions on average $(a, b)$ and parahippocampal gyrus $(c, d)$. The dotted line shows the regression line in the $\mathrm{AD}$ group. Detailed data on the correlation in each ROI were shown in Online Resource 2 for PBB3 and Online Resource 3 for PiB.

1) Difference in $\mathrm{PBB} 3$ or $\mathrm{PiB}$ accumulation between $\mathrm{AD}$ group and $\mathrm{HC}$ group.

2) Correlation between MMSE and PBB3, PiB SUVR. n.s.: not significant. 
a

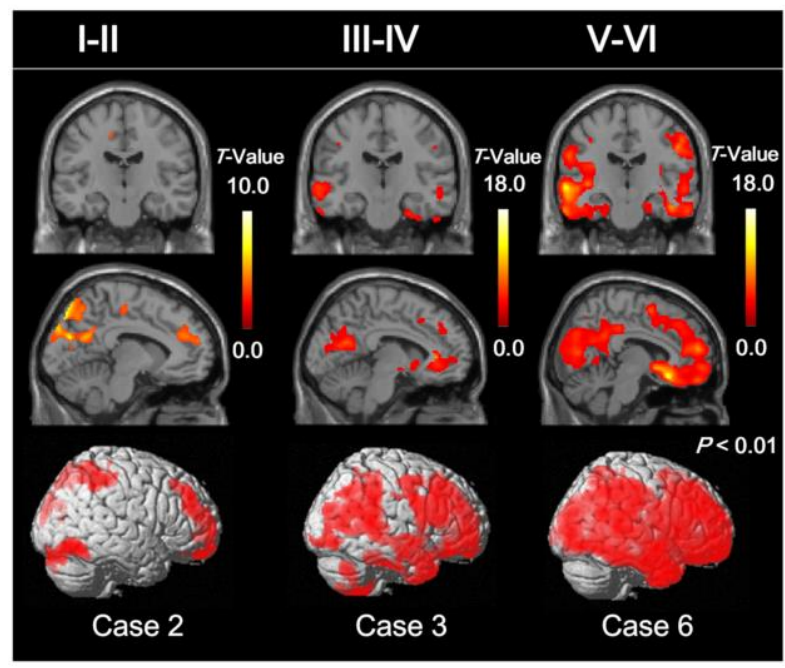

b

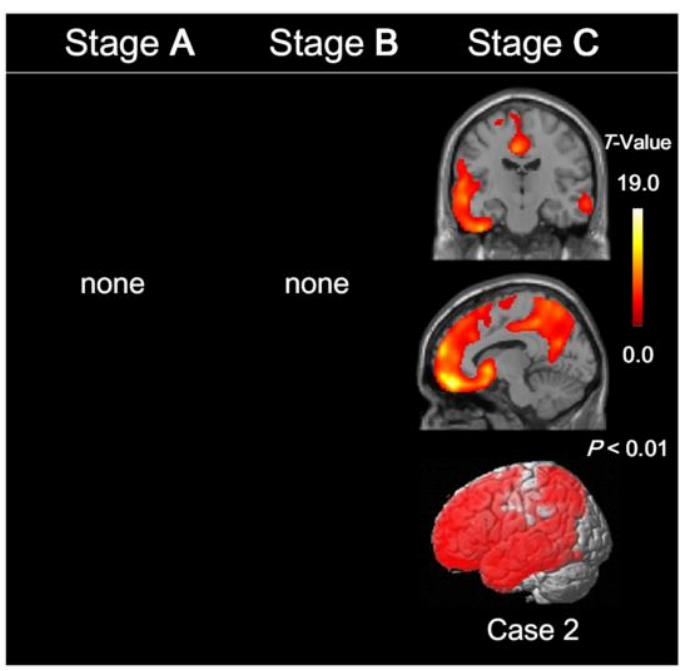

Figure 1. Representative cases for Braak's staging of NFT (a) and senile plaque (b). In each case with $\mathrm{AD}, \mathrm{PBB} 3$ (a) as well as PiB (b) accumulation was statistically compared with that of $\mathrm{HC}$ by the statistical parametric mapping method. No case was classified as stage A or stage B in Braak's staging of amyloid distribution (b). 

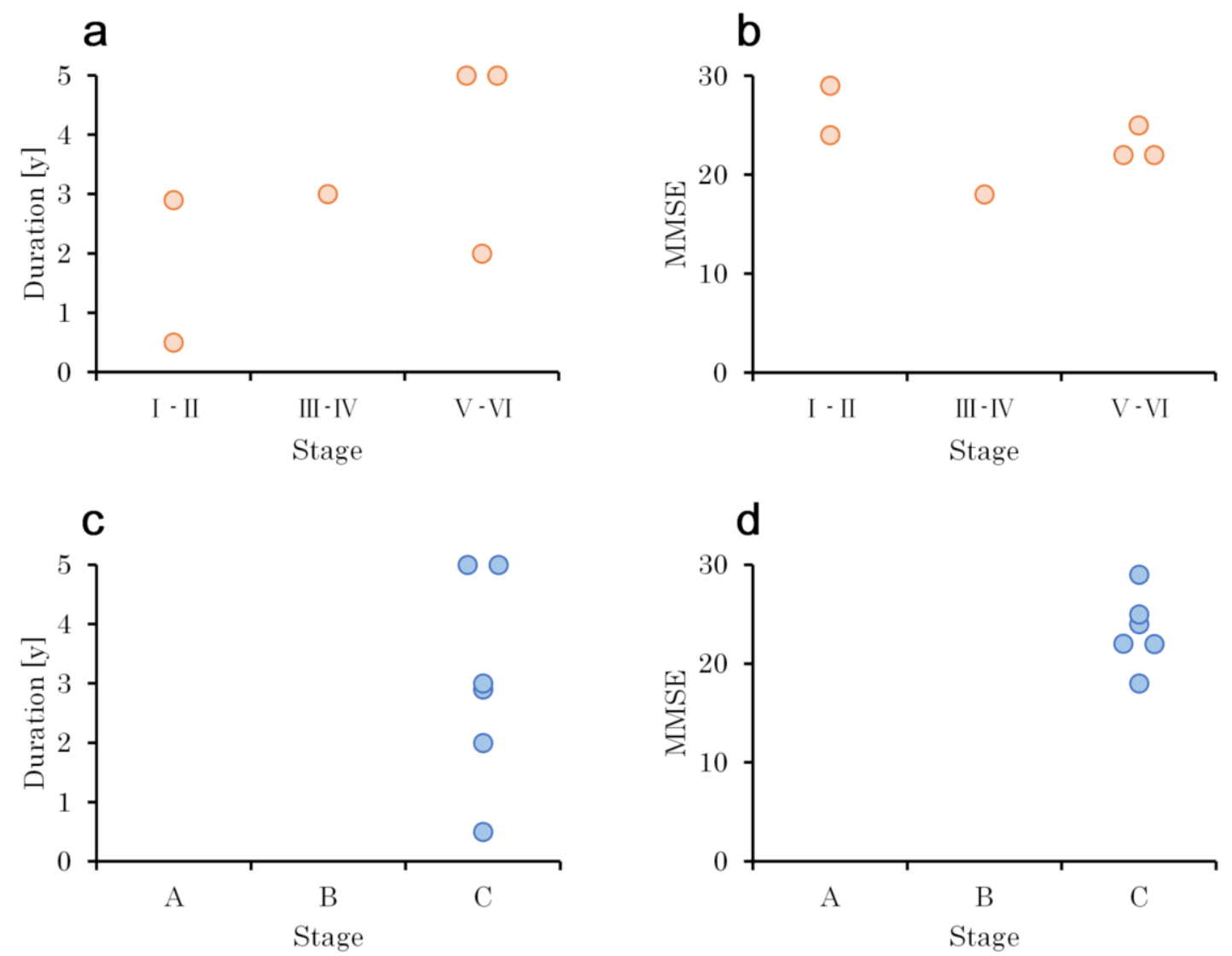

Figure 2. Disease duration and Mini-Mental State Examination (MMSE) score at each stage in patients with $\mathrm{AD}$. (a), (b) indicate neurofibrillary change staging of PBB3, (c) and (d) indicate amyloid deposits staging of PiB. 
a

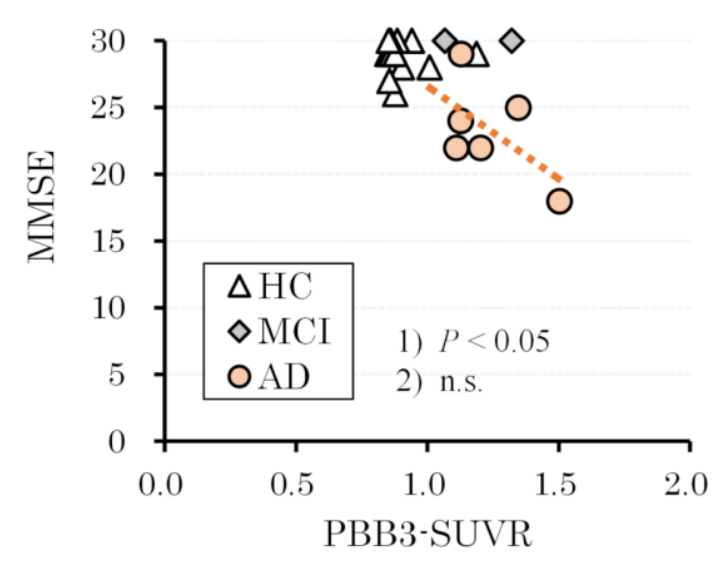

C

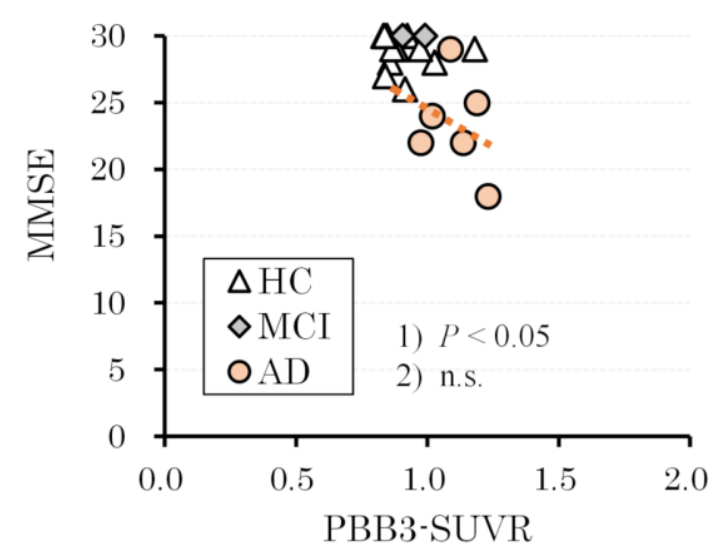

b

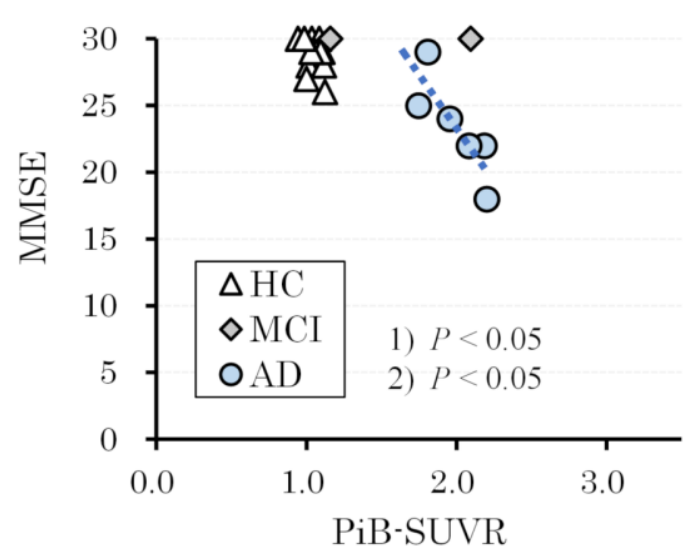

d

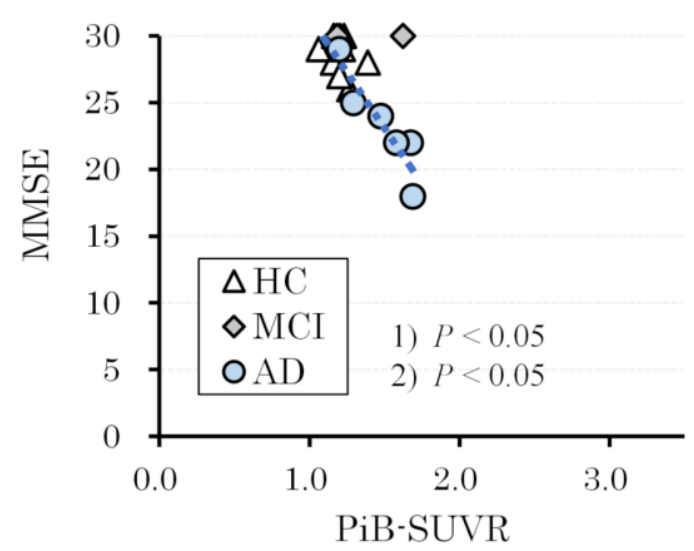

Figure 3. Scatter plots show correlation between Mini-Mental State Examination (MMSE) and PBB3 SUVR (a, c) or MMSE and $\mathrm{PiB}$ SUVR $(b, d)$ in $\mathrm{AD}$ signature regions on average $(a, b)$ and parahippocampal gyrus (c, $d$ ). The dotted line shows the regression line in the $\mathrm{AD}$ group. Detailed data on the correlation in each ROI were shown in Online Resource 2 for PBB3 and Online Resource 3 for PiB.

1) Difference in $\mathrm{PBB} 3$ or $\mathrm{PiB}$ accumulation between $\mathrm{AD}$ group and $\mathrm{HC}$ group.

2) Correlation between MMSE and PBB3, PiB SUVR. n.s.: not significant. 
Table 1. Demographic data

\begin{tabular}{|c|c|c|c|c|c|c|c|}
\hline $\begin{array}{c}\text { Subject } \\
\text { No. }\end{array}$ & Age & Gender & $\begin{array}{c}\text { Disease } \\
\text { Duration (y) }\end{array}$ & MMSE & HDS-R & $\begin{array}{l}\text { Braak } S \\
\text { PBB3 }\end{array}$ & $\begin{array}{l}\text { tage } \\
\text { PiB }\end{array}$ \\
\hline \multicolumn{8}{|c|}{ Alzheimer's Disease } \\
\hline 1 & 74 & M & 0.5 & 29 & 22 & I-II & $\mathrm{C}$ \\
\hline 2 & 85 & M & 2.9 & 24 & 19 & I-II & $\mathrm{C}$ \\
\hline 3 & 51 & M & 3.0 & 18 & 19 & III-IV & $\mathrm{C}$ \\
\hline 4 & 70 & $\mathrm{~F}$ & 5.0 & 25 & 24 & V-VI & $\mathrm{C}$ \\
\hline 5 & 78 & M & 2.0 & 22 & 21 & V-VI & $\mathrm{C}$ \\
\hline 6 & 60 & $\mathrm{~F}$ & 5.0 & 22 & 27 & V-VI & $\mathrm{C}$ \\
\hline \multicolumn{8}{|c|}{ Mild Cognitive Impairment } \\
\hline 7 & 81 & $\mathrm{~F}$ & n.a. & 30 & 30 & I-II & Negative \\
\hline 8 & 82 & M & n.a. & 30 & 30 & III-IV & $\mathrm{C}$ \\
\hline \multicolumn{8}{|c|}{ Healthy controls } \\
\hline $\mathrm{n}=12$ & $71.8 \pm 8.7^{\dagger}$ & $\mathrm{M} / \mathrm{F}=7 / 5$ & n.a. & $28.8 \pm 1.3^{\dagger}$ & $28.8 \pm 1.7^{\dagger}$ & $\begin{array}{l}\text { Age dependent } \\
\text { accumulation }\end{array}$ & Negative \\
\hline
\end{tabular}

MMSE: Mini-Mental State Examination, HDS-R: revised Hasegawa Dementia Scale n.a.: not available, $\dagger$ average \pm standard deviation 